\title{
A novel superconducting quantum interference device for biomagnetic measurements
}

\author{
ZHANG ShuLin $^{1,2^{*}}$, ZHANG GuoFeng ${ }^{1,2}$, WANG YongLiang ${ }^{1,2}$, ZENG Jia ${ }^{1,2,3}$, QIU Yang ${ }^{1,2,3}$, \\ LIU Ming ${ }^{1,2}$, KONG Xiang Yan ${ }^{1,2^{*}}$ \& XIE XiaoMing ${ }^{1,2}$
${ }^{1}$ State Key Laboratory of Functional Materials for Informatics, Shanghai Institute of Microsystem and Information Technology, Chinese Academy of Sciences, Shanghai 200050, China;
${ }^{2}$ Joint Research Laboratory on Superconductivity and Bioelectronics, Collaboration between CAS-Shanghai and FZJ, Shanghai 200050, China; \\ ${ }^{3}$ Graduate University of the Chinese Academy of Sciences, Beijing 100049, China
}

Received January 9, 2013; accepted May 24, 2013

\begin{abstract}
Superconducting Quantum Interference Device (SQUID) can provide an ultrahigh magnetic sensitivity for the biomagnetic measurements. In this paper, a SQUID bootstrap circuit (SBC) gradiometer was designed and fabricated. Using the SBC gradiometer, magnetocardiography (MCG), fetal MCG and magnetoencephalography (MEG) signals were detected in a magnetically shielded room.
\end{abstract}

SQUID bootstrap circuit (SBC), gradiometer, magnetocardiography (MCG), fetal MCG, magnetoencephalography (MEG)

Citation: $\quad$ Zhang S L, Zhang G F, Wang Y L, et al. A novel superconducting quantum interference device for biomagnetic measurements. Chin Sci Bull, 2013, 58: 2917-2919, doi: 10.1007/s11434-013-5968-y

Superconducting quantum interference devices (SQUIDs) have been widely used to detect the very weak biomagnetic fields from picotesla (pT) down to femtotesla (fT), such as human magnetoencephalography (MEG), magnetocardiography (MCG) and fetal MCG [1]. Besides the high sensitive SQUID sensors which are needed for successfully implementation of biomagnetic measurements, the other key problem is to detect such weak signals under an environment with very strong background fields. At present, different SQUID systems are employed: SQUID magnetometers for the measurements performed in super magnetically shielding rooms (MSRs) or SQUID gradiometers with different antenna figurations in moderate MSRs or unshielded environments $[2,3]$.

In this work, we report a helium-cooled gradiometer based on a novel SQUID named SQUID bootstrap circuit (SBC) [4-6]. By using the SBC gradiometer, biomagnetic measurements was performed in a moderate MSR. Besides MCG and MEG signals, fetal MCG signals were also detected.

*Corresponding authors (email: zhangs1@mail.sim.ac.cn; xykong@mail.sim.ac.cn)
SBC is a voltage-biased SQUID direct readout scheme. By increasing the SQUID flux-to-current coefficient and the dynamic resistance with two branches, the flux-to-voltage coefficient $(\partial V / \partial \Phi)_{\mathrm{SBC}}$ of SBC is enhanced to suppress the noise from the preamplifier.

Figure 1(a) shows a schematic diagram of our designed SBC. Using a voltage feedback coil of inductance $L_{2}$ and a shunt resistor $R_{\mathrm{S}}$ in series, the $(\partial V / \partial \Phi)_{\mathrm{SBC}}$ can be enhanced by increasing the dynamic resistance. In order to suppress the external magnetic noise in the SBC chip area, a dual-loop washer-SQUID of a gradiometer configuration was designed. The input coil couples external magnetic flux to the SQUID by connecting a first-order axial wire-wound antenna, as shown in Figure 1(b). The antenna has a configuration of $1: 1$ with a baseline of $50 \mathrm{~mm}$ and $18 \mathrm{~mm}$ in diameter. In order to enhance the noise suppression, a thin niobium shielding tube was used to cover the SQUID chip, as indicated in Figure 1(b).

Figure 1(c) shows the measured field spectral density of the SBC gradiometer in our MSR. The noise level was measured to be $5 \mathrm{fT} / \sqrt{\mathrm{Hz}}$ at $100 \mathrm{~Hz}$. 
(a)

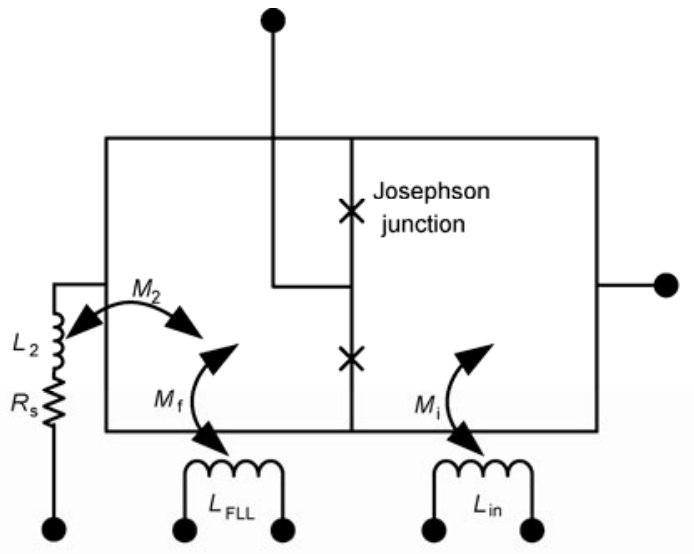

(b)
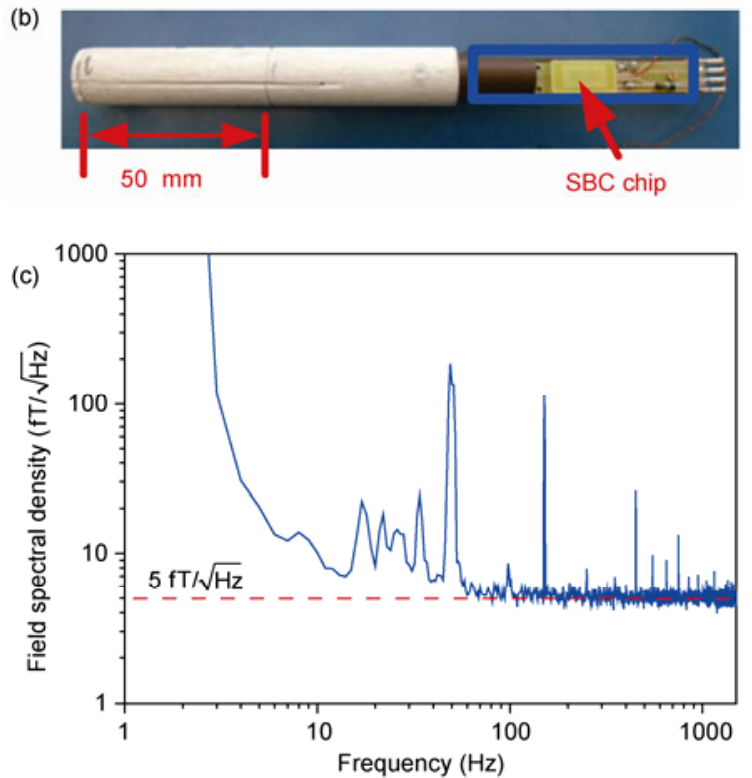

Figure 1 SBC gradiometer. (a) Schematic diagram of the designed SBC; (b) photograph of the gradiometer module; (c) noise spectrum of the SBC gradiometer.

Based on the SBC gradiometer, a biomagentic measurement system was set up in our MSR. The SBC gradiometer was mounted in a special nonmagnetic glass fiber cryostat. The distance between the lower pick-up coil of the antenna and the outside bottom of the cryostat is $16 \mathrm{~mm}$. In order to reduce the external magnetic disturbances, the data acquisition and processing system was placed outside the MSR. By using the SBC system, MCG, fetal MCG and MEG signals were detected.

The inset of Figure 2 shows the real-time MCG signals of an adult recorded with a sampling rate of $2560 \mathrm{~Hz}$. The bandwidth is $0.1-150 \mathrm{~Hz}$. By averaging data of 40 cycles, MCG signal with a signal to noise ratio of $40 \mathrm{~dB}$ was obtained. The R peak of the MCG signal was approximately 40 pT (Figure 2).

The inset of Figure 3 shows the recorded real-time fetal MCG signal with a sampling rate of $1 \mathrm{kHz}$. The gestational age of the fetus is 30 weeks. The high-frequency noise and baseline drifts were removed by combination of a $90 \mathrm{~Hz}$

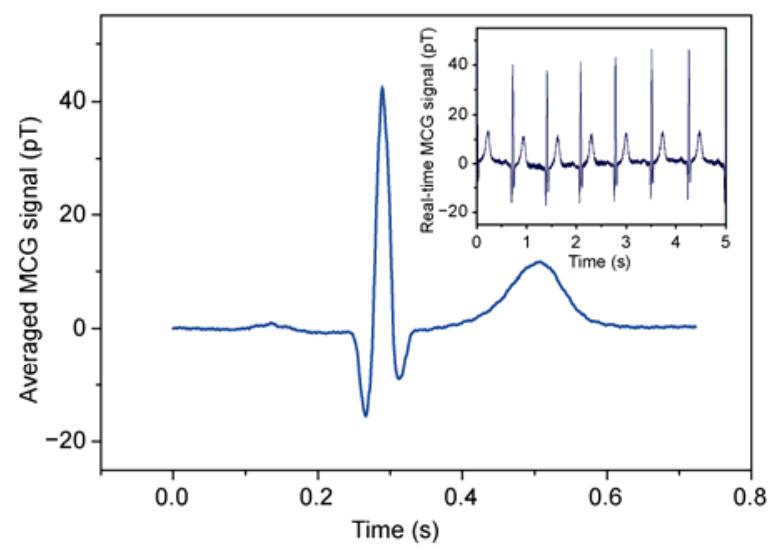

Figure 2 Averaged MCG signal of 40 cycles (inset: real-time signal).

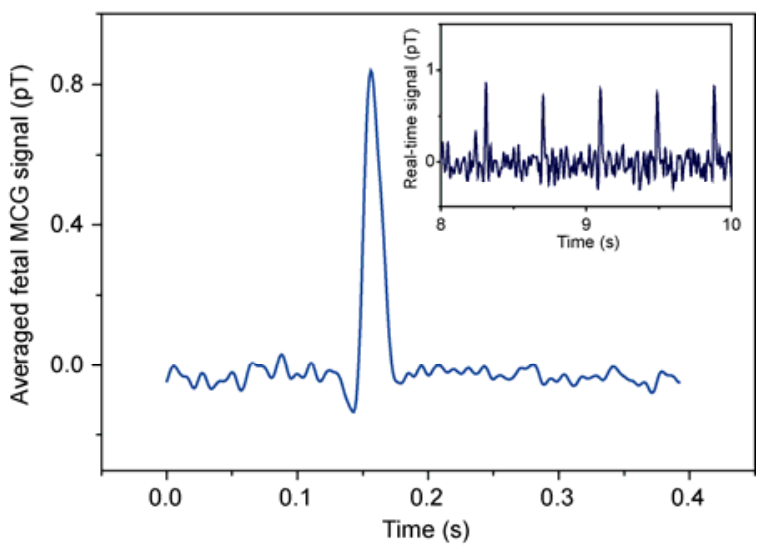

Figure 3 Averaged fetal MCG signal of 30-week gestination fetus from 36 cycles (inset: real-time signal).

low-pass FIR filter and the wavelet transformation. Figure 3 shows the averaged fetal MCG signal of 36 cycles. Comparing to the above measured adult MCG signal, the fetal MCG signal was weak and only about 0.8 pT.

For the MEG measurement, auditory evoked magnetic field was detected. The stimulus was a pure sinusoidal tone with a frequency of $1 \mathrm{kHz}$, a duration of $100 \mathrm{~ms}$ and an interval of $1.1 \mathrm{~s}$. The inset of Figure 4 shows the averaged MEG trace by 100 times with a sampling rate of $375 \mathrm{~Hz}$. High frequency noise was removed by a low-pass filter of $35 \mathrm{~Hz}$. From the trace, the prominent N100m peak can be clearly recognized [7]. The amplitude was about $0.14 \mathrm{pT}$.

From the above results, amplitudes of all the measured signals were smaller than those reported before [8-10]. For the MCG and fetal MCG signals, the smaller results attribute to the shorter gradiometer baseline of $50 \mathrm{~mm}$ [11], which is also the reason why the maternal MCG signal was not observed during the fetal MCG measurements. For the MEG signal, it is because of the non-specially designed MEG cryostat [12].

In conclusion, a SBC gradiometer with a noise level of $5 \mathrm{fT} / \sqrt{\mathrm{Hz}}$ at $100 \mathrm{~Hz}$ was developed and applied for the biomagnetic measurements. Besides the MCG and MEG 


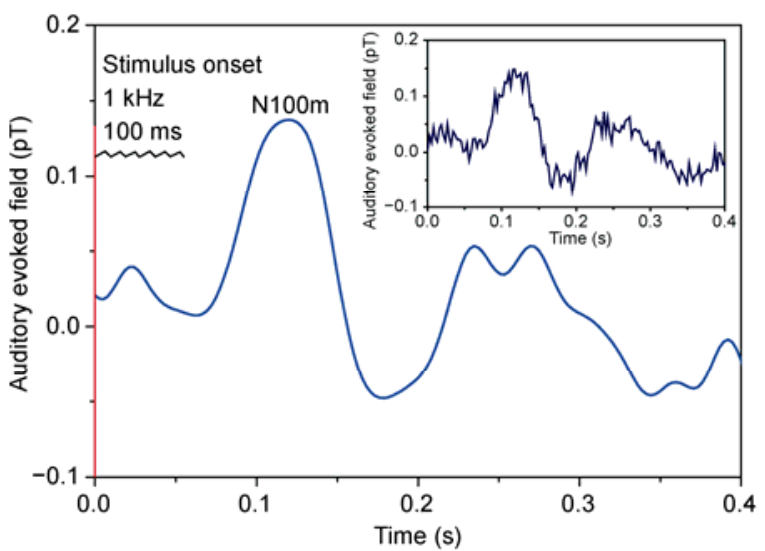

Figure 4 Auditory evoked magnetic field averaged by 100 times (inset: signal without filter).

signals, a fetal MCG signal was also measured for the first time in China. Further optimization of the SBC gradiometer and development of a multichannel biomagnetic system will be studied in the near future.

The authors thank Professor Yi Zhang from the Research Center Jülich, Germany, for his enthusiastic encouragement and fruitful discussion. This work was supported by the Knowledge Innovation Program of the Chinese Academy of Sciences (KGCX2-YW-906, KGCX2-EW-105), "Hundred Talents Program" of the Chinese Academy of Sciences and Strategic Priority Research Program (B) of the Chinese Academy of Sciences (XRD04020300).

1 Sternickel K, Braginski A I. Supercond Sci Tech, 2006, 19: S160S171

2 Pizzella V, Penna S D, Gratta C D, et al. Supercond Sci Tech, 2001, 14: R79-R114

3 Vrba J, Haid G, Lee S, et al. Clin Phys Physiol Meas, 1991, 12: 81-86

4 Xie X M, Zhang Y, Wang H W, et al. Supercond Sci Tech, 2010, 23: 065016

5 Zhang Y, Zhang G F, Wang H W, et al. IEEE Trans Appl Supercon, 2011, 21: 501-504

6 Zhang G F, Zhang Y, Zhang S L, et al. Physica C, 2012, 480: 10-13

7 Hämäläinen M, Hari R, Ilmoniemi R J, et al. Rev Mod Phys, 1999, 30: 413-497

8 Schneider S, Abraham-Fuchs K, Reichenberger H, et al. Physiol Meas, 1993, 14: A55-A60

9 Bick M, Stemickel K, Panaitov G, et al. IEEE Trans Appl Supercon, 2001, 11: 673-676

10 Comani S, Srinivasan V, Alleva G, et al. Phys Med Biol, 2007, 53: N87-N97

11 Zhang Y, Wolters N, Lomparski D, et al. IEEE Trans Appl Supercon, 2005, 15: 631-634

12 Knuutila J E T, Ahonen A I, Hämäläinen M S, et al. IEEE Trans Magn, 1993, 29: 3315-3321

Open Access This article is distributed under the terms of the Creative Commons Attribution License which permits any use, distribution, and reproduction in any medium, provided the original author(s) and source are credited. 